\title{
Performance of single-trial classifications of viewed characters using EEG waveforms
}

\section{Minoru Nakayama*}

The Centre for Research and Development of

Educational Technology (CRADLE),

Tokyo Institute of Technology,

Ookayama, Meguro,

Tokyo 152-8552, Japan

Email: nakayama@cradle.titech.ac.jp

*Corresponding author

\section{Hiroshi Abe}

Department of Human System Science,

Tokyo Institute of Technology,

Ookayama, Meguro,

Tokyo 152-8552, Japan

Email: abe@nk.cradle.titech.ac.jp

\begin{abstract}
This paper examines the possibility of classifying characters viewed by subjects using single-trial Electroencephalogram (EEG) waveforms from the frontal and occipital areas of the brain. As a training data set, Event-Related Potentials (ERPs) were calculated for each character from the first 20 trials and the remainder were assigned to a test data set. To extract features of waveforms, the regression relationship between the EEG and ERP waveforms was calculated from the training data set using the Support Vector Regression (SVR) technique. As a measure of classification performance, cross-validation rates were calculated for the test data set and they incrementally increased with the number of channels when the regression relationship was used. This result provides evidence that this procedure using the relationship between EEGs and ERPs is effective in predicting viewed characters, and that performance can be improved by a combination of waveforms across electrodes.
\end{abstract}

Keywords: visual perception; lexical perception; EEG waveforms; single trial EEG; ERP; event-related potential; Kanji perception; signal processing; SVR; support vector regression; classification; cross-validation rate; chronological analysis.

Reference to this paper should be made as follows: Nakayama, M. and Hiroshi, A. (2012) 'Performance of single-trial classifications of viewed characters using EEG waveforms', Int. J. Cognitive Biometrics, Vol. 1, No. 1, pp.10-25.

Biographical notes: Minoru Nakayama graduated from Tokyo Gakugei University in 1983 and completed the MEd programme in 1985 and received Dr. of Engineering degree from Tokyo Institute of Technology in 1990. He is presently a Professor at the Centre for Research and Development of Educational Technology (CRADLE), Tokyo Institute of Technology, Japan. His research concerns educational technology. 
Hiroshi Abe received his $\mathrm{BS}, \mathrm{MS}$ and $\mathrm{PhD}$ degrees in Engineering from Tokyo Institute of Technology, Tokyo, Japan in 2001, 2003 and 2007, respectively. $\mathrm{He}$ is presently a postdoctoral associate in the Department of Neurobiology at Yale University School of Medicine.

\section{Introduction}

Human visual perception has been studied using a chronological approach because the information being viewed is processed as from a low level to a high level (Posner and Raichle, 1994). Since we can quickly recognise most objects and make decisions, image processing requires very little time (Bacon-Macé et al., 2005). Not only visual stimuli, but lexical decisions can be made in around $500 \mathrm{~ms}$ as conventional reaction time studies show (Wagenmarkers et al., 2008).

Lexical perception has a very important role in our daily life because this process is required prior to the recognition of any message. Event-Related Potentials (ERPs) (Rugg, 1997) are often used for lexical perception analysis, as well as for the study of the image perception process. To extract an ERP waveform, waveforms of Electroencephalograms (EEGs) which record responses to stimulus need to be averaged as much as is possible. As information about decision making can be extracted from EEG waveforms, EEGs can be applied to a Brain Machine Interface (BMI) or to a Brain Computer Interface (BCI) (Blankertz et al., 2001; Devlaminck et al., 2009) using some signal processing techniques. Recently, a technique employing an fMRI as a BCI technique (Miyawaki et al., 2009) has been used to observe viewer's recognition of characters.

This suggests that there is a possibility of obtaining information about images viewed from a subject's brain activity.

Though EEGs have been used to observe the human visual perception process, EEGs are noisy data because a single stimulus point at the scalp is quite small $(5-10 \mu \mathrm{V})$. Therefore, most lexical studies employ ERPs rather than EEGs (Kutas and Federmeier, 2000) because ERPs are a well-known conventional technique for reducing noise on EEGs, in which the high time resolution of ERPs, which is the benefit of using ERPs, has been kept. The relationship between EEGs and ERPs is simple, but ERPs as a prototypical signal can be a key piece of information for the understanding of single-trial EEG waveforms. This suggests that single-trial EEGs for lexical decisions contain an ERP waveform with some artefacts. To extract the information from single-trial EEGs, some additional information such as references for the signals are often required (Parra et al., 2003). As ERPs can indicate some features of events, they may be used as a reference to extract distinct information from single-trial EEGs.

The authors have proposed a procedure to reduce these artfeacts using Support Vector Regression (SVR) (Smola and Scholkopf, 1998) to establish a relationship between single-trial EEGs and ERPs which are extracted from a small number of trials as a reference (Nakayama and Abe, 2009; Nakayama and Abe, 2010). According to previous studies, regression with Neural Network and SVR can be used to reduce noise as a nonlinear filter of time series signals (Bishop, 1995; Luo and Unbehauen, 1997; Smola and Scholkopf, 1998). The proposed processing procedure is based on this scientific evidence. This technique has been used previously to predict classes of symbols or Kanji viewed by 
subjects (Nakayama and Abe, 2009; Nakayama and Abe, 2010). The performance is insufficient, however. To improve it, the perceptual processes including local and chronological activities in the brain are examined using a combination of multiple waveforms are determined.

The following topics are addressed in this paper:

1 A signal prediction technique using a relationship which is based on the regression between EEGs and ERPs, is applied to measured EEG waveforms and used to evaluate the effectiveness of classifying a viewed character using cross validation rates.

2 The change of classification performance is compared across EEG electrodes and chronological phases.

3 The effect of combinations of electrodes on classification performance is examined by comparing the performance of each electrode when the classification is conducted using multiple electrodes.

This paper is organised as follows. First, the experimental methods and classification procedure are described in Section 2. Section 3 explains the classification performance using single-channel and single-trial EEG waveforms. Section 4 explains the classification performance using multiple channel single-trial EEG waveforms. The channel selection effectiveness and individual differences are discussed in Section 5. Finally, Section 6 concludes the paper and proposes future research work.

\section{Method}

\subsection{EEG/ERP waveforms}

\subsubsection{Kanji recognition task}

To observe EEG waveforms during the character perception process, a Kanji recognition task was conducted (Abe and Nakayama, 2006a; Abe and Nakayama, 2006b; Abe and Nakayama, 2006c). The experimental task is depicted in Figure 1(a). When a Kanji character or a symbol was flashed on a computer screen, EEG waveforms were recorded from 21 electrodes, as shown in Figure 1(b). This experiment examined the Kanji recognition process in accordance with the differences in ERP waveforms among Kanji which were known, Kanji which were unknown and symbols. The known Kanjis were selected using a Japanese lexical database (Amano and Kondo, 1999). The number of trials for each kind of stimuli, symbols, known Kanji and unknown Kanji, was 100. For each subject, a total of 200 trials were analysed. The procedure was as follows:

1 The subject clicked the left button of a mouse to start the trial.

2 A black screen which gave a random delay of 1.8-2.2 s was then presented.

3 An image of a character (either a known Kanji, an unknown Kanji, or a symbol) was presented briefly $(83 \mathrm{~ms})$.

4 The subject had to report if the image presented was a known Kanji, an unknown Kanji or a symbol, by clicking the mouse's left or right button respectively. Four subjects who were $23-25$ years old participated in this experiment. 
Figure 1 Experimental task for Kanji recognition (a) and placement of electrodes (b) (Nakayama and Abe, 2010)

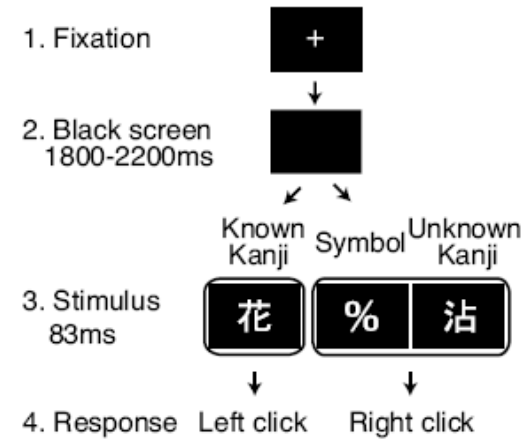

(a)

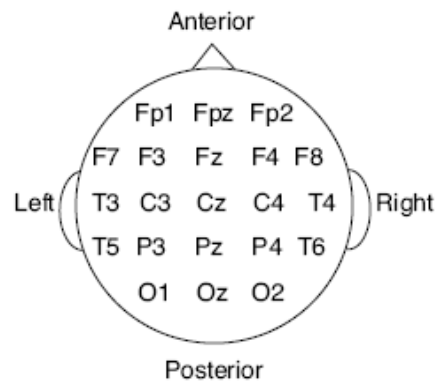

(b)

\subsubsection{EEG/ERP recording}

The EEGs were recorded from 21 scalp electrodes which included Fpz, Oz and 19 other locations, according to the international 10-20 system, using an electrocap manufactured by Electro-cap International. A ground electrode was placed on the forehead. All scalp electrodes were referenced to the subject's ear lobes. The signals were amplified and $0.5-100 \mathrm{~Hz}$ band pass filtered using a BIOTOP amplifier from NEC. The signals were stored in a PC that had a $512 \mathrm{~Hz}$ sampling rate. After being recorded, the signals were digitally filtered to reduce line noise below $40 \mathrm{~Hz}$. Trials containing an EOG amplitude of more than $70 \mu \mathrm{V}$ were removed from the subsequent analyses, because of the large artefactual influences of eye movements on EEGs (Makeig et al., 1999). Each subject's single-trial raw EEGs were recorded from every electrode, from $100 \mathrm{~ms}$ before to $1000 \mathrm{~ms}$ after stimulus onset in all correct trials. Baseline voltages were subtracted from the raw data of these EEGs. As a baseline for each trial and for each electrode, the average voltage in the preceding $100 \mathrm{~ms}$ interval before stimulus onset during the trial was used. Correct trials were trials in which the subject correctly discriminated between the characters or symbols presented.

ERP waveforms reflect the kind of characters that were shown (Abe and Nakayama, 2006a; Abe and Nakayama, 2006b; Abe and Nakayama, 2006c). Figure 2 shows an ERP extracted from the $\mathrm{Oz}$ electrode. The waveforms of all correct responses were summed up and compared with ERP waveforms of symbols and Kanji. These ERP waveforms are well smoothened using a simple moving average and show the differences in response to the stimuli. Some significant differences between symbols and Kanji were observed in P100 (at 100 ms: amplitude, symbols $>$ Kanji, $t(5)=2.61, p<0.05$ ), N170 (at 170 ms: latency, symbols $>$ Kanji, $t(5)=5.51, p<0.05)$. There is no significant difference in P250 (at $250 \mathrm{~ms}$ ), however. Mean reaction time was around $500 \mathrm{~ms}$ and the waveforms between 100 and $500 \mathrm{~ms}$ were significantly different because the recognition processes were different (Abe and Nakayama, 2006a; Abe and Nakayama, 2006c).

In this paper, we focused on EEG waveforms for symbols and known Kanji from several electrodes in the occipital and frontal area because the occipital area reflects visual perception and the frontal area reflects the decision making process. The effectiveness is evaluated using measured signals from electrodes from both areas of the brain. 
Figure 2 ERP waveforms of symbol and Kanji character stimuli from the Oz electrode

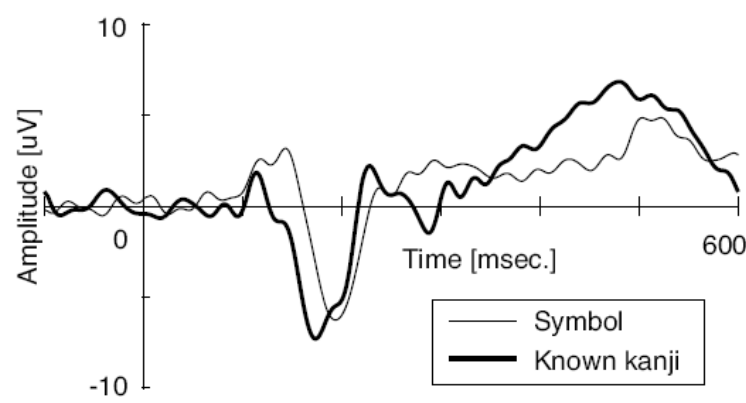

\subsection{Classification of single-trial EEGS}

\subsubsection{Data pre-processing}

The classification performance for single-trial EEG waveforms from several electrodes was evaluated using the diagram in Figure 3. Therefore, only trials with correct responses were selected. Rates of correct selection averaged over 90\% (Abe and Nakayama, 2006a). All EEG waveforms for each subject were smoothened using a simple moving average in advance, to permit the application of some signal processing. The first 20 trials, which showed both symbols and Kanji, were used to create a training data set for each subject. A test data set was then created using the remainder of the data. Since EEG measuring equipment requires some calibration before being used, the some of first few trials may be assigned to a preparation stage.

Figure 3 EEG signal processing diagram
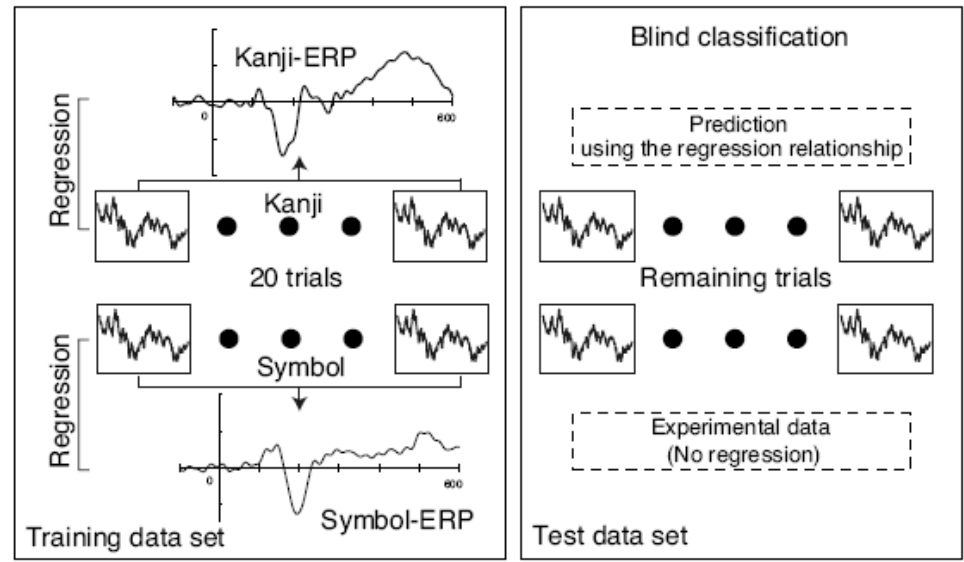

\subsubsection{Signal transformation with ERP references}

A relationship between EEGs and ERPs has been created from the training data set, which consists of 20 trials which show both symbols and Kanji (total: 40 trials). Both 
features of ERP waveforms in response to symbols and Kanji are extracted, because the features of EEGs while stimuli are being viewed should be emphasised using a common relationship. The relationship containing common features is established as follows.

First, the local ERPs for symbols and Kanji were extracted from the training data sets of each subject. ERP is defined as a simple summation of all EEG waveforms from 20 trials as shown in Figure 3. Both ERPs and single-trial EEGs are illustrated with a common time course because ERP is extracted from the summation of EEGs during the complete time course. Therefore, 20 corresponding mapping pairs of ERP and a singletrial EEG with a common time course for symbols or Kanji can be created using the relationship between ERPs and single trial EEGs. Here, a regression process is used when there is the possibility of predicting ERP from EEG waveforms because both contain common feature of the waveforms. The relationship is mathematically formulated as a regression function across each pair. Since the regression prediction from a singletrial EEG waveform may reflect both ERP features of symbols and Kanji, an appropriate signal in response to ERP waveforms from observed EEG signals is predicted. When the regression function SVR technique is applied to a set of data of potentials of a single-trial EEG, a potential of ERP is created. In this paper, a set of data consisted of ten potentials in series. The detailed processing is indicated in the mathematical procedure. This regression training procedure was conducted on a training data set, which consisted of EEGs of both symbols and Kanji for each subject. After training, the regression function can be applied to blinded waveforms in a test data set which also consists of EEGs of both symbols and Kanji for each subject. The function may emphasise the features of waveforms in response to symbols and Kanji from EEG waveforms.

The mathematical procedures are described hereafter.

All data were based on raw signal data. The regression function $f$ was created for the training data set. Here, $x_{i}$ is defined as EEG potentials and $y_{i}$ is defined as an ERP which sums up EEG waveforms of 20 trials in the training data set. The pairs $\left(x_{i}, y_{i}\right)$ are created for both symbols and Kanji in the training data set. The regression processing was conducted for every set of ten EEG potentials $\left(\mathrm{x}_{\mathrm{k}}=\left(x_{k}-9, \ldots, x_{k}\right)\right)$ and an ERP potential $y_{k}$ at the time position $k$, then the function as $f$ was trained with all training data including data for both symbols and Kanji.

For the regression prediction, the estimated ERP $\hat{y}_{k}$ for the empirical potential $y_{k}$ at the time position $k$ is reproduced from time series data samples comprising ten input vectors $\mathrm{x}_{\mathrm{k}}$, so that $\mathrm{x}_{\mathrm{k}}=\left(x_{k}-9, \ldots, x_{k}\right), \hat{y}_{k}=f\left(\mathrm{x}_{\mathrm{k}}\right)$. The parameters of SVR were given as a standard deviation for Gaussian kernel and as a width of error pipe (epsilon: eps $=0.5$ ) using SVMTorch (Collobert, 2000; Collobert and Bengio, 2001).

The trained regression function is applied to EEG waveforms in the test data set. A set of ten input vectors $\mathrm{x}_{\mathrm{k}}$ is created for each trial in the test data set as well. Then the transformed waveform can be defined as $f\left(\mathrm{x}_{\mathrm{k}}\right)$. This transformed waveform is different from the original empirical potential. According to the hypothesis, the transformed waveform emphasised one of two features for symbols and Kanji. As the experimental procedure suggests, all waveforms in the trials respond to the viewed character class $t$. Therefore the relationship can be noted as $\left(t, f\left(\mathrm{x}_{\mathrm{k}}\right)\right)$ for each trial, in both the training and test data sets.

This signal processing is called 'REG', and the empirical data set without the processing is called 'NO-REG'. 


\subsubsection{Classification of EEG waveforms}

In trials, EEG waveforms reacted when characters were viewed by subjects. In this section, another procedure to estimate viewed characters from both the transformed and empirical EEG waveforms is described.

All trial data contain significant response periods, which were controlled to be from $100-150 \mathrm{~ms}$ (for 26 data points) to $100-500 \mathrm{~ms}$ (for 205 data points) long. The duration was extended step by step, by $100 \mathrm{~ms}$, up to $500 \mathrm{~ms}$. From $500 \mathrm{~ms}$ to $800 \mathrm{~ms}$ (for 153 data points) data were collected because the response decision had been made. Classification labels for symbols or Kanji were given to each trial data set. The relationship between a trial data set and classification label can be noted as follows.

Here, $x_{i}$ is defined as an EEG potential and $\hat{y}_{i}$ is defined as the transformed potential, $t$ is defined as the stimulus given and the data set comprises $N$ input vectors $\mathrm{x}=\left(x_{1}, \ldots, x_{N}\right)$ as 'NO-REG' and $\hat{y}=\left(\hat{y}_{1}, \cdots, \hat{y}_{N}\right)$ as 'REG', with corresponding target values: $t \in\{-1$ : symbol, $+1:$ Kanji $\}$. The acquired data can be noted as $(\mathrm{x}, t)$ and $(\hat{y}, t)$ for each trial. A sign function, based on the SVM function, is defined as $G$ using the Gaussian kernel. For every interval, the parameters for the Gaussian kernel were optimised using a software tool (Chang and Lin, 2001). Classification was conducted using SVM with Gaussian kernel, such as LIBSVM (Chang and Lin, 2001).

When the sign function has been created from the training data set (the left side of Figure 3), the function can be applied to the test data set and can predict a viewed character class $\hat{t}$ which is given as $\hat{t}=G_{x}(\mathrm{x})$ for 'NO-REG' or $\hat{t}=G_{\hat{y}}(\hat{\mathrm{y}})$ for 'REG' from waveforms of each trial (the right side of Figure 3 ). The classification performance has been reported, but performance may depend on the quality of the training data set (Nakayama and Abe, 2010), however. To assess the effectiveness of the transformation using the relationship between ERP and EEG waveforms, cross validation rates of the test data set are calculated across various conditions. The rate indicates that the data has the ability to be classified (Stork et al., 2001).

\section{Results of classification using a single channel}

The ability to classify the test data set was assessed as a fivefold cross-validation rate using LIBSVM (Chang and Lin, 2001). In this procedure, the test data set was randomly divided into five blocks and the classification performance was assessed. The rates were calculated for each subject and the mean rates across the two classification procedures were summarised.

Generally, the classification performance of a test data is evaluated set using a trained classifier with a set of training data. In this paper, the main purpose is the evaluation of the effectiveness of applying the regression process and the transformation between ERP and EEG waveforms using the relationship. Therefore, that the difference in the possibility of classification was evaluated using fivefold cross-validation rates for the test data set which was applied to the transformation.

\subsection{Cross-validation rate for $\mathrm{Oz}$ and $\mathrm{Fz}$}

The results for the $\mathrm{Oz}$ electrode of all subjects are summarised in Figure 4. The horizontal axis shows the duration and the vertical axis shows the cross-validation rate. The 'NO-REG' shows the results using raw observation data. With the extension of 
the duration, the rate increases gradually and the highest rate is during a duration of 100-500 ms. Using a non-parametric test (Shiba and Watabe, 1976), this rate is significant when the rate is over $0.58(p<0.05)$, but other rates are not significant except in a condition with a duration of 500-800 ms. When predicted EEG waveforms were applied to classifications such as 'REG', all rates were significant except for conditions with a duration of 100-150 ms and 100-200 ms. In particular, data for a duration of 100-300 ms became significant when the estimated EEG waveforms were applied using the regression function. This duration includes P100, N170 and P250, so that predictions using the regression relationship between EEG and ERP may emphasise the differences in waveforms. This also suggests that the data for a duration of 100-200 ms is insufficient for its classification, however.

Figure 4 Cross-validation rate $(\mathrm{Oz})$

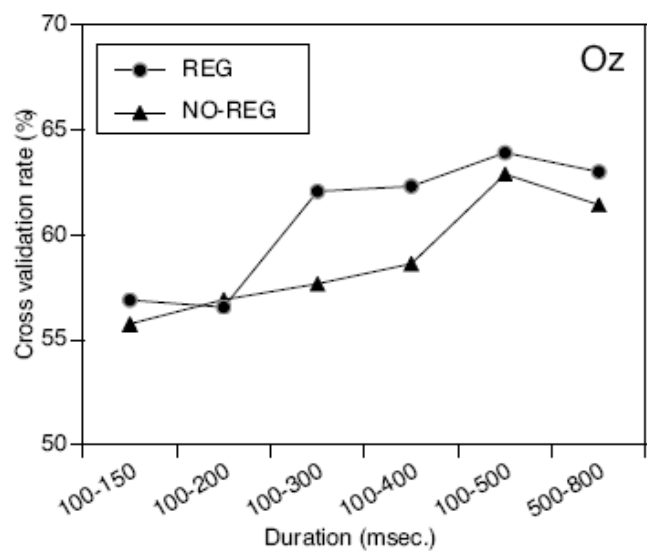

The results for $\mathrm{Fz}$ electrodes of all subjects are summarised in Figure 5. The highest performance classification was for a duration of 100-500 ms across the three processing conditions. For this duration, 'REG' is higher than 'NO-REG'. The performance with predictions using the regression relationship is almost always higher than the performance with raw observed data across all durations. For durations up to $400 \mathrm{~ms}$ all rates are not significant, however the rate with predictions is almost always higher.

Figure 5 Cross-validation rate $(\mathrm{Fz})$

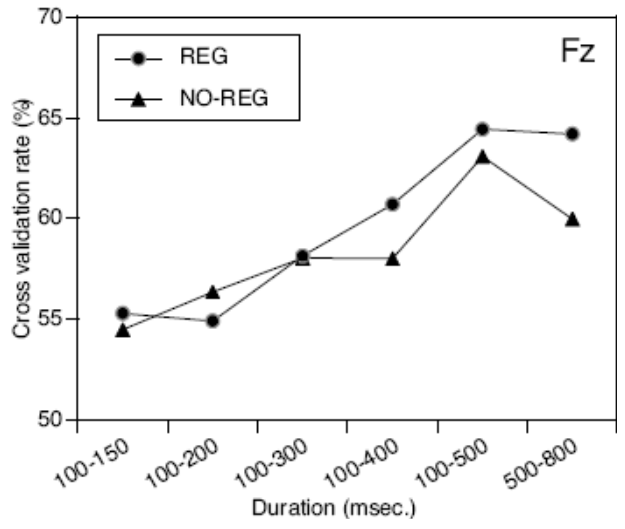




\subsection{Chronological difference}

In comparing the results for $\mathrm{Oz}$ and $\mathrm{Fz}$, the duration for the highest rates are different. For the Oz classification, predicted waveforms up to $300 \mathrm{~ms}$ are sufficient and predicted waveforms up to $500 \mathrm{~ms}$ are required for Fz classification. Both classification results suggest that EEG waveforms before the button was pressed to make a selection (at around $500 \mathrm{~ms}$ ) reflect the viewed characters specifically. From this, it can be determined that single-trial EEG waveforms contain significant information in response to viewed characters.

In the general visual information processing theory, the retinal image is mapped in the primary visual cortex, which is located in the occipital area (Posner and Raichle, 1994). During the decision task, some activation may be observed in the frontal cortex, which is located in the frontal area. Some time is needed to recognise characters which are shown, resulting in the highest peak of the cross-validation rate being delayed until perception has occurred. The greater delay in perception by $\mathrm{Fz}$ as compared to $\mathrm{Oz}$ coincides with the chronological model, but the measured time of the delay seems longer than in theory. Therefore, some factors may influence the cross-validation rates in addition to the influence of the image processing pathway.

These results suggest that predictions using the relationship between EEGs and ERPs are effective.

\section{Results of classification using multiple channels}

According to the above results, the performance was limited when the classification was conducted using single channel waveforms. In this chapter, the data is extended to waveforms of multiple channels, such as the data of two or three channels combined.

\subsection{Cross-validation rate using both $\mathrm{Oz}$ and $\mathrm{Fz}$}

In the above section, the classification performance was determined using single-channel signals for $\mathrm{Oz}$ and $\mathrm{Fz}$. To determine the effectiveness of using multiple channels, a test data set was created by merging two data sets for $\mathrm{Oz}$ and $\mathrm{Fz}$, such as $\left(\mathrm{x}_{O z}, t\right)$ and $\left(\mathrm{x}_{F z}, t\right)$. Because they have been measured simultaneously and the target values are common as $t$ $\in\{-1:$ symbol, $+1:$ Kanji $\}$, the merged data set can be noted as $\left(\mathrm{z}_{O z+F z}, t\right)$, where $\mathrm{z}_{O z+F z}=$ $\left(\mathrm{x}_{O z}+\mathrm{x}_{F z}\right)$, or $\mathrm{z}_{O z+F z}=\left(\mathrm{x}_{O z, 1}, \ldots, \mathrm{x}_{O z, N}, \mathrm{x}_{F z, 1}, \ldots, \mathrm{x}_{F z, N}\right)$. The number of dimensions for $\mathrm{z}_{O z+F z}$ is twice the number of dimensions for a single-channel signal. Therefore, a new prediction function $H$ is also defined using the Gaussian Kernel, as $\hat{t}=H_{O z+F z}\left(Z_{O z+F z}\right)$. The classification performance is assessed using cross-validation rates.

According to the results, we have confirmed that both signals contain significant information about the characters being viewed. As the combination of those signals might provide more distinct information, an improvement in classification performance was expected. The cross-validation rates of classification performance using both features of $\mathrm{Oz}$ and $\mathrm{Fz}$ of all subjects are calculated and summarised in Figure 6, using the same format. 
Figure 6 Cross validation rate $(\mathrm{Fz}+\mathrm{Oz})$

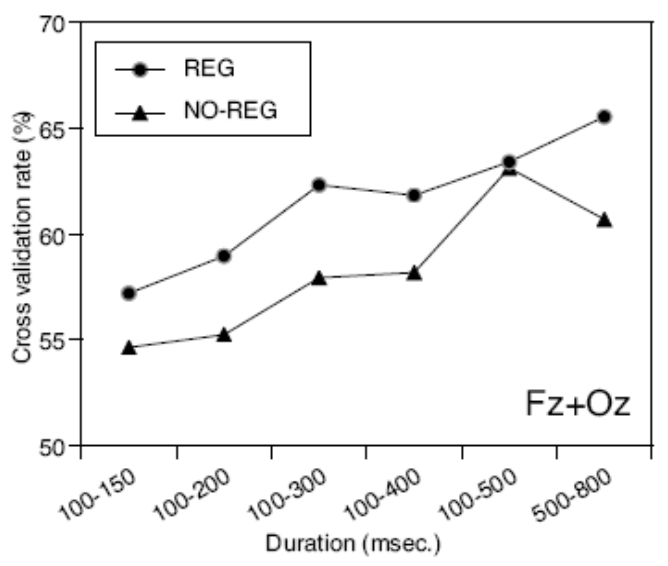

The rates increase along with the duration. The rates for 'REG' are higher than the rates for 'NO-REG', and the rates at 500-800 ms are the highest. The performance in the area of $100-300 \mathrm{~ms}$ and $100-400 \mathrm{~ms}$ shows the same level of Oz (Figure 4), and the performance in the area of 100-500 ms and 500-800 ms also shows the same level of Fz (Figure 5). Though a mutual effect was expected using the combination of $\mathrm{Oz}$ and $\mathrm{Fz}$, the effectiveness of using a combination was unclear. As a possible reason, the duration phases for the highest performances were different between $\mathrm{Oz}$ and $\mathrm{Fz}$. In the previous section, differences in the highest performances for duration between $\mathrm{Oz}$ and $\mathrm{Fz}$ were obtained. Therefore, performance could not have been due to a mutual effect.

\subsection{Performance using occipital channels}

To synchronise the duration of signals, EEG waveforms for occipital channels were analysed. The activity in the occipital area may relate directly to the recognition of viewed characters. According to the placement of electrodes in Figure 1, O1 and $\mathrm{O} 2$ were selected as additional EEG information about $\mathrm{Oz}$ for classifying viewed characters.

Figure 7 Cross-validation rate (O2)

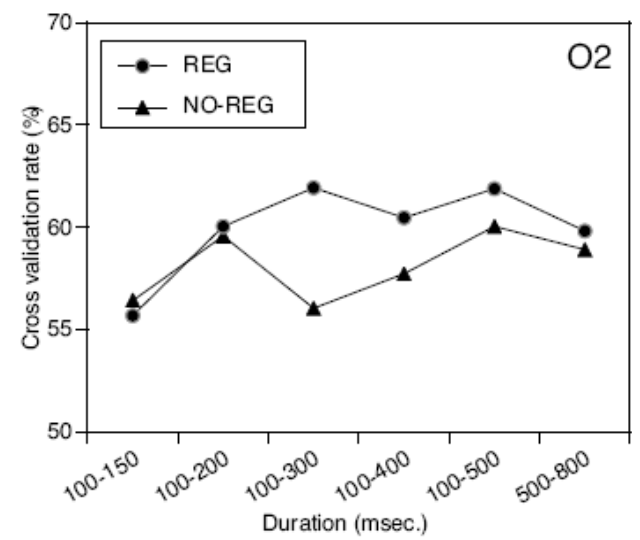


First, the effectiveness of classifying signals with ERP references for $\mathrm{O} 1$ and $\mathrm{O} 2$ was determined as previously with the $\mathrm{Oz}$ electrode. The cross-validation rates for $\mathrm{O} 2$ of all subjects are summarised in Figure 8. The rates for ' $R E G$ ' are higher than the ones for 'NO-REG' when the duration is from $100-300 \mathrm{~ms}$ to $100-500 \mathrm{~ms}$. The effectiveness of the ERP reference is also determined at the $\mathrm{O} 2$ electrode, and the phases where performance has improved have the same duration as with the $\mathrm{Oz}$ electrode.

Figure 8 Cross-validation rate $(\mathrm{O} 2+\mathrm{Oz})$

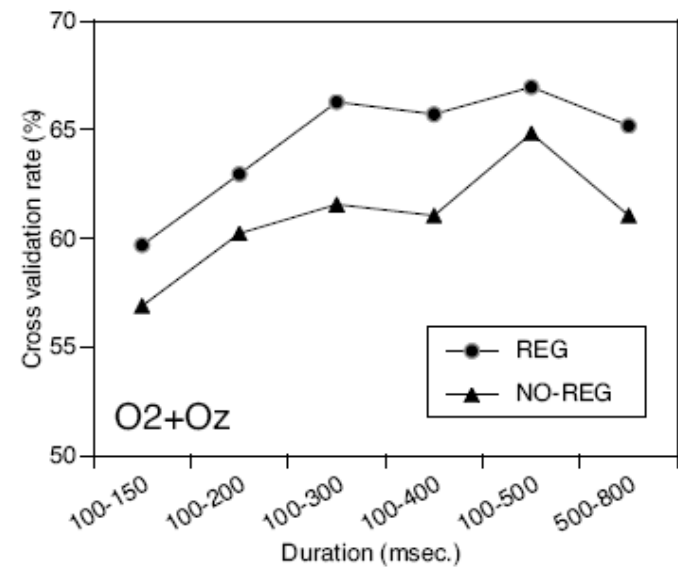

The performance for the combination of $\mathrm{O} 2+\mathrm{Oz}$ was calculated using the same procedure. Again, the input vectors are noted such as $\mathrm{z}_{O 2+O z}=\left(\mathrm{x}_{O 2}, \mathrm{x}_{O z}\right)$, and the prediction function is defined as $\hat{t}=H_{O 2+O z}\left(Z_{O 2+O z}\right)$.

The cross-validation rates for $\mathrm{O} 2+\mathrm{Oz}$ of all subjects are summarised in Figure 8 . The effectiveness of classifying signals with ERP references is obvious from 100-300 ms to 100-500 ms because the rates for 'REG' are higher than in the 'NO-REG' condition. These rates also are higher than the rates for $\mathrm{O} 2$ or $\mathrm{Oz}$ by themselves, in the duration from $100-300 \mathrm{~ms}$ to $100-500 \mathrm{~ms}$ and $500-800 \mathrm{~ms}$. In this combination, the mutual effectiveness is confirmed. The mutual effectiveness for $\mathrm{O} 2$ and $\mathrm{Oz}$ is higher than it is for $\mathrm{Oz}$ and Fz. This suggests that the channel combination is an important issue for the improvement of performance.

\section{Discussion}

\subsection{Channel selection issue}

Since the combined effectiveness of electrodes in the occipital area was confirmed, the performance of a combination of three channels in the occipital area was evaluated. Using the same procedure, triple sets of input vectors are combined as $\mathrm{Z}_{O 1+O 2+O z}=$ $\left(\mathrm{x}_{O 1}, \mathrm{x}_{O 2}, \mathrm{x}_{O z}\right)$, and the prediction function is defined as $\hat{t}=H_{O 1+O 2+O z}\left(\mathrm{z}_{O 1+O 2+O z}\right)$. 
The results of cross-validation rates for $\mathrm{O} 1+\mathrm{O} 2+\mathrm{Oz}$ of all subjects are shown in Figure 9. All phases are synchronised across the results of three electrodes, and the rates of the combinations show the same tendencies. The effectiveness of classifying signals with ERP references is obvious from $100-300 \mathrm{~ms}$ to $100-500 \mathrm{~ms}$ because the rates for 'REG' are also higher than the rates for the 'NO-REG' condition. However, the overall performance is at the same level as the rates for $\mathrm{O} 2+\mathrm{Oz}$. According to the hypothesis, the performance may increase monotonically when synchronised waveforms are combined, because synchronised responses may be emphasised using combinations of multiple EEG electrodes. This result may suggest the possibility that performance improvements are limited when the number of channels in the same area increases during classification, however.

Figure 9 Cross-validation rate $(\mathrm{O} 1+\mathrm{O} 2+\mathrm{Oz})$

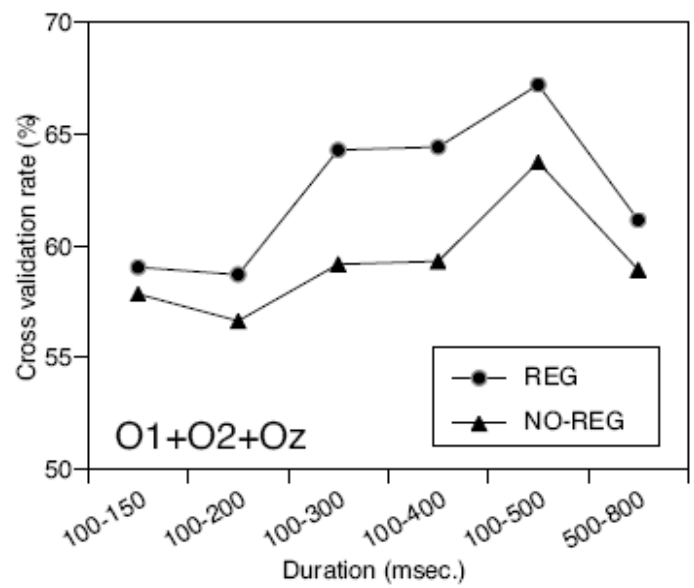

\subsection{Individual difference}

One possible reason for the small improvement in the number of channels is the subject's individual differences. Generally, there are various differences between individuals and sometimes these differences affect overall performance. In this experiment, four subjects were participants.

One subject (Sub A) clearly shows the effectiveness of processing with ERP references. Figure 10 shows the performance which consists of the number of channels with and without ERP references. The greatest performance increase is with the number of channels and by the processing of 'REG' as ERP references. The rates at 100-500 ms are above $75 \%$, and the performance improvement has been clearly obtained.

Another subject (Sub D) does not show good performance because all rates stay at the level of chance except ' $\mathrm{REG}(\mathrm{O} 1+\mathrm{O} 2+\mathrm{Oz})$ '. The performance improvement of the rates are determined, however, when the number of channels is increased and signal processing with ERP references are provided. The rates for 'NO-REG' stay at the level of chance, near $50 \%$ and the discriminant ability of signals may not be high. The baselines and improvements in classification performance are individually different; therefore the overall improvement in performance across subjects is limited. 
Figure 10 Cross-validation rate (Sub A)

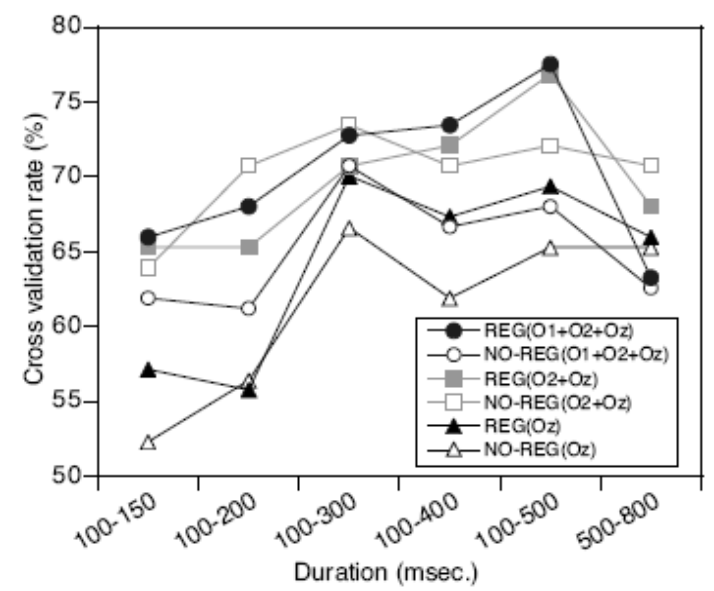

Figure 11 Cross-validation rate (Sub D)

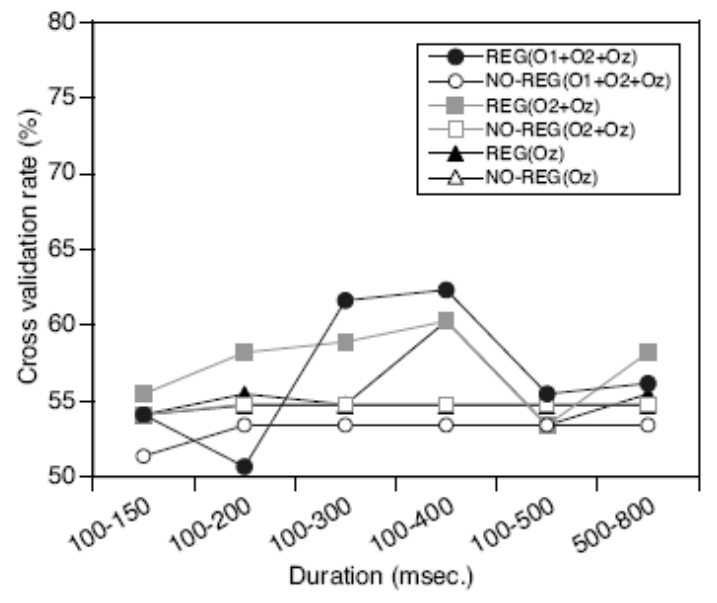

Both individual results indicate a significant improvement of the rates when signal processing with ERP references and a combination of EEG waveforms from multiple electrodes are applied to classification. The synchronised electrodes can be extracted using a popular EEG signal processing technique, such as Independent Component Analysis (ICA), which can display the common spatial patterns of both electrodes and their waveform activities simultaneously (Abe and Nakayama, 2006b). Therefore, an appropriate set of electrodes can be selected easily. Again, the effectiveness of EEG waveform processing with ERP references has been confirmed. In this paper, the first 20 trials were assigned as a prototype of EEG waveforms for the proposed procedure. If they could not extract and emphasise the features of the waveform, signal processing with ERP references made insufficient transformations. The better validated procedure of gathering prototype waveforms in response to the visual stimuli should be considered as a means to improve classification performance. 


\section{Conclusion}

In this paper, a new procedure for estimating viewed characters observed by subjects is proposed using a transformation of single-trial EEG waveforms of the human brain. The performance is evaluated using a cross validation rate for the test data of each subject. To improve the performance, classification was conducted using a single channel from specific electrodes in the frontal and occipital areas, and also using various combinations of electrodes. The following points were realised:

1 According to the proposed model, the relationship between EEGs and ERPs were created mathematically, using the SVR technique for several electrodes in the frontal and occipital areas. The ability to classify signals using cross-validation rates were calculated for some duration. The rates increased from a duration of 100-300 ms to $100-500 \mathrm{~ms}$ and $500-800 \mathrm{~ms}$. The effectiveness of the transformation using the relationship was confirmed.

2 In comparing the cross-validation rates between frontal and occipital areas, the rates for the occipital area were improved from a duration of $100-300 \mathrm{~ms}$ to $100-500 \mathrm{~ms}$ but the rates for the frontal area were significant only from a duration of 100-500 ms. This suggests that there is a phase difference between areas which reflects the time course of lexical processing.

3 The rates increased when the classification was conducted using a combination of multiple electrodes which came from same area, such as $\mathrm{O} 1+\mathrm{O} 2+\mathrm{Oz}$. The effectiveness of this was also confirmed individually.

These results provide evidence that effective transformation of single-trial EEG waveforms can be established using the relationship between EEGs and ERPs as a regression between the two, and classification performance can be improved by a combination of electrodes placed in carefully considered positions.

To produce a sample for the practical use of this procedure, a practice session needs to be conducted in order to calibrate and establish a mathematical relationship between EEGs and ERPs. This session produces a set of training data prior to the measuring session. After that, predictions can be conducted using transformed EEG signals.

To further improve the classification procedure, other signal processing techniques such as applying a low-pass filter or down-sampling for ERP should be tried and evaluated. Also, an extension of the multiple classifications of viewed characters and the development of possible applications using this technique will be subjects for our further study.

On the other hand, the baselines and improvements in classification performance are individually different, therefore this characteristic can be deployed to a biometric system. This possibility should be determined in our further study.

\section{Acknowledgements}

The authors would like to thank that IEEE for granting to us the permission to reprint portions of the described IEEE copyrighted conference proceedings paper. Figure 1 and some explanations about the figures appear as in Nakayama and Abe (2010). 


\section{References}

Abe, H. and Nakayama, M. (2006a) 'Event-related potential study of Kanji perception process', The Journal of the Institute of Image Information and Television Engineers, Vol. 60, pp.397-404.

Abe, H. and Nakayama, M. (2006b) 'An ICA approach for extracting task-evoked components from EEG signals', Proceedings of the 2006 International Symposium on Intelligent Signal Processing and Communication System (ISPACS2006), pp.862-865.

Abe, H. and Nakayama, M. (2006c) 'An investigation into Kanji character discrimination process from EEG signals', Enformatika, Transaction on Engineering, Computing and Technology, Vol. 18, pp.13-18.

Amano, S. and Kondo, K. (1999) NTT database series Nihongo-no Goitokusei (Lexical properties of Japanese), Sanseido, Tokyo, Japan.

Bacon-Macé, N., Macé, M.J., Fabre-Thorpe, M. and Thorpe, S.J. (2005) 'The time course of visual processing: backward masking and natural scene categorisation', Vision Research, Vol. 45, No. 11, pp.1459-1469.

Bishop, C.M. (1995) Neural Networks for Pattern Recognition, Oxford University Press, Oxford, UK.

Blankertz, B., Curio, G. and Muller, K. (2001) 'Classifying single trial EEG: towards brain computer interfacing', Proceedings of the Neural Information Processing Symposium (NIPS), pp.157-164.

Chang, C-C. and Lin, C-J. (2001) LIBSVM: A Library for Support Vector Machines. Available online at: http://www.csie.ntu.edu.tw/ cjlin/libsvm/

Collobert, R. (2000) SVMTorch II. Available online at: http://www.idiap.ch/

Collobert, R. and Bengio, S. (2001) 'SVMTorch: support vector machines for large-scale regression problems', Journal of Machine Learning Research, Vol. 1, pp.143-160.

Devlaminck, D., Wyns, B., Boullart, L., Sntens, P. and Otte, G. (2009) 'Brain-computer interfaces: from theory to practice', Proceedings of the17th European Symposium on Artificial Neural Networks (ESANN), pp.415-424.

Kutas, M. and Federmeier, K.D. (2000) 'Electrophysiology reveals semantic memory use in language comprehension', Trends in Cognitive Science, Vol. 4, No. 12, pp.463-470.

Luo, F-L. and Unbehauen, R. (1997) Applied Neural Networks for Signal Processing, Cambridge University Press, New York, USA.

Makeig, S., Westerfield, M., Jung, T.P., Covington, J.T.J., Sejnowski, T.J. and Courchesne, E. (1999) 'Functionally independent components of the late positive event-related potential during visual spatial attention', Journal of Neuroscience, Vol. 19, pp.2665-2680.

Miyawaki, Y., Uchida, H., Yamashita, O., Sato, M., Morio, H., Tanabe, H., Sadato, N. and Kamitani, Y. (2009) 'Visual image reconstruction from human brain activity using a combination of multiscale local image decoders', Neuron, Vol. 60, pp.915-929.

Nakayama, M. and Abe, H. (2009) 'The possibility of single-trial classification of viewed characters using EEG waveforms', Proceedings of the 17th European Symposium on Artificial Neural Networks (ESANN), pp.449-454.

Nakayama, M. and Abe, H. (2010) 'Feasibility of using single-channel EEG waveforms for single-trial classification of viewed characters', Proceeding of the International Conference on Information Society (i-Society 2010), pp.228-233.

Parra, L., Alvino, C., Tang, A., Pearlmutter, B., Yeung, N., Osman, A. and Sajda, P. (2003) 'Single-trial detection in EEG and MEG: keeping it linear', Neurocomputing, Vols. 52-54, pp.177-183.

Posner, M.I. and Raichle, M.E. (1994) Images of Mind, Scientific American Library, USA.

Rugg, M.D. (1997) Cognitive Neuroscience, Psychology Press, UK. 
Shiba, Y. and Watabe, H. (1976) Toukeiteki Houhou II (Stastistical Method II), Shinyosha, Tokyo, Japan.

Smola, A.J. and Scholkopf, B. (1998) A Tutorial on Support Vector Regression, NeuroCOLT2 Technical Report Series (NC2-TR-1998-030). Available online at: http://www.neurocolt.com

Stork, D.G.R., Duda, O. and Hart, P.E. (2001) Pattern Classification, 2nd ed., John Wiley \& Sons.

Wagenmarkers, E-J., Ratcliff, R., Gomez, P. and McKoon, G. (2008) 'A diffusion model account of criterion shifts in the lexical decision task', Journal of Memory and Language, Vol. 58, pp.140-159. 